\section{Edad de menarquia y ascendencia indígena. Un estudio poblacional en Chile}

\author{
XIMENA OSSA ${ }^{1,2, a}$, PATRICIA BUSTOS 3 , \\ SERGIO MUÑOZ ${ }^{1,2, b}$, HUGO AMIGO $^{3, \mathrm{c}}$
}

\section{Age at menarche and indigenous ancestry. A population study in Chile}

Background: The age at menarche may influence decisively health and disease in women. It also indicates the beginning of the reproductive period and, as a consequence, the possibility of biological continuity for the human species. Genetic and environmental determinants define the age of menarche and can explain differences found among different populations. Aim: To determine the age at menarche among adolescents with different levels of indigenous descent (parental indigenous surnames), considering the effect of socioeconomic and demographic factors. Material and Methods: An observational study of historic cohorts of 8.624 girls from the Araucania Region (central-southern Chile) was carried out. Data were collected by health professionals using a previously validated questionnaire. Occurrence of menarche was estimated through survival analysis and compared between groups (according to indigenous parental surnames) adjusted for parents' income and educational level and provenance (rural/urban). Results: Estimated median age of menarche was 151 months (95\% CI: 150-151). In female with four indigenous surnames, menarche occurred two months later than girls without indigenous surnames and with two indigenous surnames $(p<0,001)$. In girls whose parents had lowest level of schooling, the difference increased to eight months later $(p<0,005)$. Conclusions: Age at menarche in the group with higher indigenous descent is later even if socio-economic conditions remain stable. Genetic factors might play an important role, however conditions of vulnerability can influence and further delay the onset of reproductive competency.

(Rev Med Chile 2012; 140: 1035-1042).

Key words: Factors; Groups; Menarche; Socioeconomic factors.

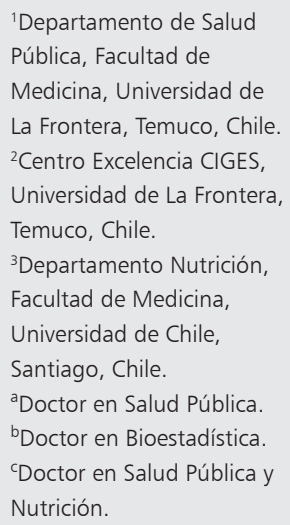

Fuentes de financiamiento: Fondo de Desarrollo Científico y Tecnológico (FONDECYT) Proyecto No 1060884 y Fondo de Mejoramiento de la Calidad de la Educación Superior (MECESUP) Proyecto FRO003

Recibido el 22 de diciembre de 2011, aceptado el 9 de mayo de 2012.

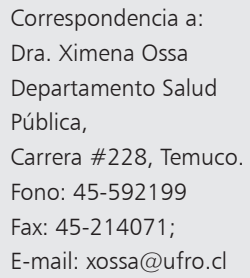

L a edad de la menarquia es una variable antropológica, sicológica y biológica que puede influir en forma determinante en algunos estados de salud-enfermedad de las mujeres. Marca, además, el comienzo de la competencia reproductiva y, como consecuencia, la posibilidad de la continuidad biológica para la especie humana. Determinantes genéticos y ambientales definen la edad de la menarquia y pueden explicar las diferencias encontradas entre diferentes grupos étnicos ${ }^{1-4}$

Desde un enfoque evolutivo, la selección natural favorece a individuos que optimizan sus com- petencias para procrear y traspasar la información genética a las nuevas generaciones, produciéndose un juego adaptativo entre las características de vida de los individuos y el medioambiente. Estas características involucran patrones y fases de crecimiento y competencias reproductivas dentro de las cuales se encuentra la menarquia ${ }^{5}$.

En Brasil y Colombia se han publicado edades de menarquia para población indígena más tempranas que lo descrito para la población general. Algunos autores postulan que la edad temprana de menarquia en algunas poblaciones indígenas puede deberse, desde un contexto ecológico, a 
una adaptación para facilitar las condiciones reproductivas en un medioambiente adverso, donde las expectativas de vida son bajas ${ }^{6,7}$. Otros estudios señalan edades más tardías en población aborigen y especialmente en lugares rurales, atribuyéndose esto a causas genéticas, escaso desarrollo y mayor vulnerabilidad social ${ }^{8-10}$.

En Chile, los últimos estudios publicados señalan una edad para la menarquia de 12,7 años en adolescentes de Santiago, pero los estudios sobre menarquia en población indígena han sido limitados a muestras no representativas y no concluyentes en sus resultados ${ }^{11-14}$.

La menarquia temprana es un factor de riesgo para algunos tipos de cáncer y otras enfermedades crónicas no transmisibles ${ }^{15-18}$, por lo que debiera constituir una preocupación en salud pública. Por otro lado, la menarquia temprana se asocia con sobrepeso en período pre-puberal y, también en el período de la adolescencia y adultez, aunque esta última asociación es controversial ${ }^{19-21}$. En Chile, se ha evidenciado diferencias significativas en la prevalencia de obesidad en mujeres de distinto nivel educacional ${ }^{22}$, lo que muestra la necesidad de estudiar en profundidad todos los determinantes que pudieran intervenir en este fenómeno, especialmente en los grupos vulnerables.

Este estudio constituyó la primera fase de un proyecto que midió el crecimiento en etapas puberales de adolescentes de diferente origen étnico. El objetivo de este trabajo fue conocer la edad de menarquia en adolescentes con distinta ascendencia indígena (número de apellidos mapuches) considerando el efecto de factores socioeconómicos y demográficos.

\section{Diseño de estudio y población}

Estudio observacional de cohortes históricas, en las que se determinó en forma retrospectiva la edad de menarquia de 8.624 adolescentes ( 8 y 16 años) que asistían a escuelas públicas de la Región de La Araucanía (zona centro-sur de Chile). Estos establecimientos fueron seleccionados aleatoriamente en 24 de las 32 comunas de la Región y corresponden al 80\% de las escuelas con mayor concentración indígena. Al interior de cada escuela fueron encuestadas todas las niñas que voluntariamente aceptaron responder la encuesta, lo que constituyó el $98 \%$ de las asistentes a estos establecimientos educacionales.

\section{Recolección de datos}

La información se obtuvo mediante un cuestionario con preguntas abiertas, previamente validado, contestado por las niñas en la sala de clase. Este proceso fue guiado y supervisado por una profesional matrona y acompañado por una profesora del establecimiento. Se estimuló el recuerdo de la edad de la menarquia con fechas importantes como cumpleaños, fiestas y vacaciones. En una sub-muestra de 274 niñas (131 indígenas y 143 no indígenas) se corroboró con la madre o cuidadora el dato entregado por la participante. Las preguntas abarcaron: fecha de nacimiento, mes, año y edad de la menarquia (método de status $q u o$ ), apellidos parentales, ubicación geográfica de la escuela, escolaridad de la madre o, en su defecto del padre; finalmente, el ingreso familiar fue estimado de datos obtenidos acerca de la ocupación del jefe de hogar en la encuesta y confirmada por la profesora respectiva. Estas ocupaciones se clasificaron por grupo (Clasificación Internacional Uniforme de Ocupaciones, CIUO-88 ${ }^{23}$ y luego categorizadas en niveles utilizando como referencia el de las remuneraciones medias mensuales reales según grupo ocupacional para el país publicada por el Instituto Nacional de Estadística de Chile ${ }^{24}$.

\section{Análisis de datos}

Se procedió al análisis descriptivo del grupo de estudio según número de apellidos parentales indígena (desde cero a cuatro apellidos de origen mapuche). Para tal efecto se trabajó con un listado de los apellidos de origen mapuche que fue revisado y validado por especialistas en la lengua mapuche ${ }^{25}$.

La edad de menarquia se calculó en meses desde el mes y año de nacimiento hasta el mes y año en que ocurrió el evento. Las niñas que no habían experimentado su menarquia contribuyeron con los tiempos desde el mes y año de nacimiento hasta el momento de la aplicación del cuestionario.

Se realizó una estimación de la mediana de la edad de la menarquia a través de análisis de sobrevida y comparación de la distribución de los tiempos de ocurrencia de la menarquia (curvas de sobrevida) entre los diferentes grupos de ascendencia indígena utilizando test de Wilcoxon (recomendado para distribuciones de sobrevida cuyas funciones de riesgo no son proporcionales en el tiempo y para diferencias de sobrevida tempranas) con ajuste por método de Bonferroni 
Edad de la menarquia y ascendencia indígena - X. Ossa et al

para comparaciones múltiples definidas a priori $(\mathrm{p}<0,005)^{26}$. Posteriormente, fueron evaluados los tiempos de ocurrencia de menarquia ajustados por nivel socioeconómico y origen urbano o rural.

\section{Aspectos éticos}

Este estudio contó con la aprobación del Comité de Ética de la Facultad de Medicina de la Universidad de Chile. Se obtuvo el asentimiento informado de las niñas previa aplicación del cuestionario, respaldado por las pautas éticas internacionales para la investigación biomédica en seres humanos.

\section{Resultados}

De los 8.624 cuestionarios aplicados, la muestra quedó conformada por 8.495 niñas por rechazo de 129 encuestas incompletas $(1,52 \%$, debido a deficiencias en los datos de los apellidos parentales o imprecisiones en la fecha de nacimiento o de menarquia). Entre las características socio-demográficas (Tabla 1), se observa una correlación inversa entre el número de apellidos indígenas y la escolaridad de la madre y el nivel de ingresos del jefe de hogar $(\mathrm{p}<0,001)$; a mayor número de apellidos mapuche existe menor nivel de educación y de ingresos. Tanto en relación con la escolaridad e ingresos de los progenitores, hubo una proporción de niñas que desconocía el dato
(13,2\% y $6,7 \%$ respectivamente). Las escuelas fueron predominantemente urbanas, encontrándose directa correlación entre mayor número de apellidos mapuche y asistencia a escuelas rurales $(\mathrm{p}<0,001)$.

De todas las niñas, 39,2\% no habían tenido su menarquia al momento del estudio. La mediana estimada para la edad de menarquia, considerando los datos censurados, fue de 151 meses (IC 95\% 150-151), esto es 12,58 años. La correlación entre el dato entregado por la niña y su madre o cuidadora en la sub-muestra fue de $100 \%{ }^{14}$. La mediana para edad de menarquia fue similar en los grupos con cero y con dos apellidos mapuches, pero ambos tuvieron una diferencia significativa con las niñas con cuatro apellidos mapuches en quienes la mediana fue dos meses más tardía $(\mathrm{p}<0,001)$ (Tabla 2). Realizando una comparación en la proporción de ocurrencia de menarquia (curvas de sobrevida) por grupos de apellidos, de acuerdo a la edad, se observa que la proporción de niñas que había experimentado su menarquia antes de los 10 años fue baja (menos de 2\%); a los 11 años, esta proporción se eleva a 9\%, con una mayor proporción de niñas sin apellidos mapuches (11\%) y una menor para aquellas con cuatro apellidos mapuches (7\%). Para todas las niñas con 13 y más años, el porcentaje de experimentar la menarquia fue sobre el $60 \%$, sin diferencias por etnia. La proporción de adolescentes con menarquia más tardía fue similar en los grupos: menos de $2 \%$ la

Tabla 1. Características socio-demográficas según número de apellidos indígenas

\begin{tabular}{|c|c|c|c|c|c|c|c|c|c|c|}
\hline \multirow[t]{2}{*}{ Variable } & \multicolumn{2}{|c|}{$\begin{array}{l}\text { Sin apellido } \\
\text { mapuche }\end{array}$} & \multicolumn{2}{|c|}{$\begin{array}{l}1 \text { apellido } \\
\text { mapuche }\end{array}$} & \multicolumn{2}{|c|}{$\begin{array}{l}2 \text { apellidos } \\
\text { mapuches }\end{array}$} & \multicolumn{2}{|c|}{$\begin{array}{l}3 \text { apellidos } \\
\text { mapuches }\end{array}$} & \multicolumn{2}{|c|}{$\begin{array}{l}4 \text { apellidos } \\
\text { mapuches }\end{array}$} \\
\hline & $\mathbf{n}$ & $\%$ & $\mathbf{n}$ & $\%$ & $\mathbf{n}$ & $\%$ & $\mathbf{n}$ & $\%$ & $\mathbf{n}$ & $\%$ \\
\hline \multicolumn{11}{|c|}{ Educación de los padres* } \\
\hline Básica incompleta & 97 & 2,5 & 38 & 3,1 & 72 & 8,0 & 69 & 10,2 & 85 & 13,7 \\
\hline Básica completa & 847 & 21,5 & 379 & 30,6 & 374 & 41,3 & 363 & 53,6 & 348 & 56,0 \\
\hline E. Media & 2.492 & 63,4 & 720 & 58,2 & 412 & 45,5 & 227 & 33,5 & 172 & 27,7 \\
\hline E. Superior & 496 & 12,6 & 101 & 8,2 & 47 & 5,2 & 18 & 2,7 & 16 & 2,6 \\
\hline \multicolumn{11}{|l|}{ Ingresos familiares** } \\
\hline Sin ingresos & 397 & 9,0 & 152 & 10,9 & 158 & 15,8 & 80 & 11,2 & 78 & 11,6 \\
\hline Bajos ingresos & 789 & 17,9 & 330 & 23,8 & 335 & 33,5 & 336 & 47,1 & 348 & 51,8 \\
\hline Medio-bajo & 2.780 & 63,1 & 811 & 58,4 & 458 & 45,8 & 276 & 38,7 & 230 & 34,2 \\
\hline Medio-alto & 437 & 9,9 & 96 & 6,9 & 49 & 4,9 & 21 & 3,0 & 16 & 2,4 \\
\hline \multicolumn{11}{|l|}{ Escuela*** } \\
\hline Rural & 183 & 4,4 & 133 & 9,2 & 161 & 15,3 & 189 & 25,0 & 189 & 27,0 \\
\hline Urbana & 4.349 & 95,6 & 1.319 & 90,8 & 894 & 84,7 & 566 & 75,0 & 512 & 73,0 \\
\hline
\end{tabular}

${ }^{*}$ Rho Spearman $=-0,33, p=<0,001 .{ }^{*}$ Rho Spearman $=-0,24, p=<0,001 .{ }^{* *}$ Rho Spearman $=0,25, p=<0,001$. 
Edad de la menarquia y ascendencia indígena - X. Ossa et al

Tabla 2. Edad de la menarquia según número de apellidos mapuches

\begin{tabular}{|cccccc|}
\hline $\mathbf{n}$, de apellidos mapuches & $\mathbf{n}$ & $\mathbf{\%}$ & Mediana & Error Std & IC $\mathbf{9 5 \%}$ \\
\hline 0 & 4.531 & 53,3 & 150 & 0,24 & 150 a 151 \\
1 & 1.453 & 17,1 & 151 & 0,27 & 150 a 153 \\
2 & 1.055 & 12,4 & 150 & 0,23 & 149 a 151 \\
3 & 755 & 8,9 & 151 & 0,23 & 149 a 152 \\
\hline 4 & 701 & 8,3 & 152 & 0,22 & 150 a 153 \\
\hline Total & 8.495 & 100 & 151 & 0,24 & 150 a 151 \\
\hline
\end{tabular}

Test de Wilcoxon $X_{(4)}^{2}=18,2(p=0,001)$.

Tabla 3. Proporción de ocurrencia de menarquia por edad y número de apellidos mapuches (Análisis de sobrevida)

\begin{tabular}{|c|c|c|c|c|c|c|}
\hline \multirow[t]{2}{*}{ Edad en años } & \multirow{2}{*}{$\begin{array}{c}\text { Número que entran } \\
\text { al intervalo } \\
\mathbf{n}\end{array}$} & \multicolumn{5}{|c|}{ Número de apellidos mapuches } \\
\hline & & $\begin{array}{c}0 \\
\%\end{array}$ & $\begin{array}{c}1 \\
\%\end{array}$ & $\begin{array}{l}2 \\
\%\end{array}$ & $\begin{array}{l}3 \\
\%\end{array}$ & $\begin{array}{l}4 \\
\%\end{array}$ \\
\hline 8 & 8.495 & 0,0 & 0,0 & 0,0 & 0,0 & 0,0 \\
\hline 9 & 8.390 & 0,2 & 0,1 & 0,1 & 0 & 0,1 \\
\hline 10 & 7.774 & 1,8 & 1,2 & 1,6 & 1,8 & 1,2 \\
\hline 11 & 6.403 & 11,6 & 7,6 & 10,9 & 7,7 & 7,2 \\
\hline 12 & 4.185 & 34,0 & 31,4 & 32,4 & 33,8 & 27,7 \\
\hline 13 & 1.784 & 66,8 & 62,9 & 68,5 & 66,4 & 64,5 \\
\hline 14 & 418 & 90,2 & 87,0 & 91,5 & 91,0 & 90,1 \\
\hline 15 & 56 & 98,2 & 97,4 & 98,4 & 98,1 & 99,2 \\
\hline 16 & 6 & 99,5 & 99,0 & 100 & 100 & 100 \\
\hline 17 & - & 100 & 100 & - & - & - \\
\hline
\end{tabular}

Diferencias significativas entre los grupos 0 y 1; 0 y 4; 2 y 4 (Test de Wilcoxon con ajuste Bonferroni, $p<0,005$ ).

habían tenido después de su cumpleaños número quince (Tabla 3 ).

Se encontraron diferencias significativas en la distribución de las curvas de ocurrencia de menarquia entre los grupos con: cero y un apellido mapuches (Wilcoxon $\chi_{(1)}^{2}=8,1 \mathrm{p}=0,004$ ); cero y cuatro apellidos mapuches (Wilcoxon $\chi_{(1)}^{2}=11,6$ $\mathrm{p}<0,001)$; y dos y cuatro apellidos mapuches (Wilcoxon $\chi_{(1)}^{2}=8,2 \mathrm{p}=0,004$ ).

Cuando la muestra fue dividida por niveles de educación parental, se confirmó que existen diferencias significativas en la edad de menarquia en el grupo con menor nivel educacional, ocurriendo a los 147 meses en estudiantes sin apellidos mapuches y retrasándose hasta los 155 meses en aquellas con cuatro apellidos mapuches, es decir, ocho meses más tarde (Wilcoxon $\chi_{(1)}^{2}=8,2, \mathrm{p}=0,004$ ), manteniéndose esta diferencia al ajustar por procedencia urbano/rural. En el resto de las categorías, las diferencias por etnia no fueron significativas.

\section{Discusión}

En este trabajo, la edad de menarquia en las adolescentes no indígenas es más temprana que en las niñas con cuatro apellidos indígenas y a su vez en el grupo de mayor ascendencia indígena (cuatro apellidos mapuches) la edad de menarquia es significativamente más tardía que los demás grupos, 
incluso cuando se controla por condiciones socioeconómicas. Sin embargo, estas diferencias no necesariamente deben ser atribuidas a etnicidad, sino más bien a la participación de un grupo de factores que incluyen carga genética/étnica, nivel socio-económico y estado nutricional, entre otros.

En algunos estudios de comunidades indígenas en América Latina, la edad de menarquia se encontró por sobre los 13 años $8,10,27,28$ a diferencia del grupo con mayor ascendencia indígena en este estudio que bordea los 12,7 años. Es probable que tanto las diferencias en edad de menarquia entre los grupos como la diferencia con niñas indígenas de otros países tenga relación con las condiciones socioeconómicas respectivas. El Índice de Desarrollo Humano (IDH) para la población indígena de la región de La Araucanía está entre los más bajos del país lo que condiciona una situación de inequidad importante ${ }^{29}$ y esto podría explicar la diferencia en la edad de menarquia entre los grupos de mayor y menor ascendencia indígena en la región. En el grupo de niñas sin apellidos mapuches, los padres tienen mayor nivel educacional y de ingresos, lo cual también puede ser interpretado como mejores condiciones de vida (proxy) permitiendo expresar en ellas el potencial biológico. Algunos estudios han demostrado que entre los niveles de población con mejores condiciones socio-económicas, la menarquia se presenta a edades más tempranas debido a mejor nutrición y condiciones sanitarias de la población ${ }^{1,30-32}$. De hecho, Thomas y colaboradores sugieren que en una escala global, la menarquia aparece más temprano en países con mejores expectativas de vida ${ }^{33}$.

Por otro lado, el IDH en la población indígena de la Región es mayor que aquellos observados en otras comunidades indígenas en América Latina ${ }^{34}$. La diferencia en la edad de la menarquia, especialmente entre los grupos sin apellidos indígena y cuatro apellidos indígena, aunque significativa, no es tan marcada como ocurre con otras poblaciones indígenas del continente, por lo que se puede inferir que el grupo local se encuentra en mejores condiciones socioeconómicas que sus congéneres de otros países, lo que les permite el adelanto de la menarquia ${ }^{27,35}$.

Otro de los factores que puede explicar una edad de menarquia más temprana en las niñas indígenas es la alta prevalencia de exceso de peso que ha sido observada en todos los grupos de edad, incluida la población escolar en Chile ${ }^{36}$, con- dición que se ha ido concentrando en los niveles socioeconómicos bajos sobre todo en mujeres ${ }^{22,37}$. De acuerdo a estudios realizados tanto en población chilena como extranjera, el exceso de peso puede contribuir a una maduración sexual más temprana ${ }^{12,14,37,38}$. Estudios previos realizados en la Región de La Araucanía verificaron que había un alta prevalencia de obesidad en niños mapuches al ingreso a la escuela, condición que puede estar influyendo en que el retraso en la edad de menarquia no sea tan marcado en este grupo étnico ${ }^{39,40}$. Existe evidencia que en mujeres mapuches, la menarquia ha tendido a aparecer en forma más tardía que en aquellas mujeres no indígenas ${ }^{41}$. En una investigación realizada por nuestro grupo, con una sub-muestra de adolescentes participantes del presente estudio que incorporó mediciones antropométricas ${ }^{14}$, se demostró en el modelo final ajustando por escolaridad de la madre, que el sobrepeso y la obesidad determina significativamente reducción de la edad de menarquia y que el factor étnico aunque retrasa esta edad no alcanza significación estadística $(\mathrm{p}=0,081)$. Por estos resultados, es posible suponer que el factor genético puede estar regulando esta situación sobre todo en aquellas niñas que no presentan sobrepeso u obesidad encontrándose en ellas edades de menarquia más tardías que influyan en los resultados del grupo indígena ${ }^{42,43}$.

Por muchos años, la región de La Araucanía ha mostrado los indicadores socioeconómicos más bajos del país, no obstante, éstos han ido mejorando en los últimos años ${ }^{44,45}$ y por lo tanto, es razonable esperar que la edad de menarquia, como marcador biológico del mejoramiento de las condiciones medioambientales, también sufra modificaciones. Algunos estudios indican que la edad de menarquia en Chile estaría estacionaria desde la década de los $1970-79^{46}$, sin embargo, otra investigación realizada en la Región de La Araucanía muestra una tendencia secular hacia una menarquia más temprana en las últimas décadas sin diferenciación étnica ${ }^{43}$. Lo anterior, en conjunto con los resultados de esta investigación, deja abierta la posibilidad de que la edad de menarquia aún no se estabilice, sobre todo en poblaciones que están todavía en proceso de mejoramiento de sus condiciones de vida. Este fenómeno, en conjunto con otros factores medioambientales tanto previos como posteriores a la menarquia, puede influir en la aparición de enfermedades crónicas 
no transmisibles que requieren de mayor estudio en los grupos étnicos vulnerables.

Entre las debilidades de este estudio, cabe mencionar que la edad de menarquia fue obtenida en forma retrospectiva por lo que es un dato afecto al sesgo de memoria. Sin embargo, el intervalo ocurrido entre el evento y la recolección de información fue relativamente corto y en la sub-muestra se estimó una correlación alta con el dato entregado por la madre o cuidadora por lo que se estima un riesgo de sesgo atenuado. También podría considerarse una debilidad no haber considerado una muestra representativa de todas las comunas de la IX Región, sin embargo, esto no fue realizado de esta forma porque había 8 comunas en que la concentración de la población indígena era muy baja. Es necesario destacar que en ambos tipos de comunas (seleccionadas y no seleccionadas) el índice de desarrollo humano (IDH) era similar, con cifras que van entre 0,57 y 0,76 con una escala de 0 a 1 , siendo el promedio nacional de 0,73 (metodología para el cálculo de IDH regional y comunal 2005 especial para Chile $)^{47}$. Entre las fortalezas cabe destacar que se utilizó un cuestionario validado, aplicado por profesionales calificados en salud reproductiva, con la colaboración de madres y profesoras. También es una fortaleza el haber trabajado con una muestra suficientemente grande de niñas que asisten a escuelas públicas de la Región de La Araucanía, lo que confiere representatividad de la etnia Mapuche que habita en la Región.

Agradecimientos: Nuestros agradecimientos a las matronas Elba Contreras y Claudia Fernández por su intensa y prolija labor, así como también a los Directores de Salud Municipal de la región de La Araucanía y Directores de Escuelas y Liceos que participaron en esta investigación por su estrecha colaboración.

\section{Referencias}

1. Junqueira Do Lago M, Faerstein E, De Souza Lopes C, Werneck GL. Family socio-economic background modified secular trends in age at menarche: evidence from the Pró -Saúde Study (Rio de Janeiro, Brazil). Ann Hum Biol 2003; 30: 347-52.

2. Long JR, Xu H, Zaho LJ, Shen H, Liu YJ, Xiong DH et al. The oestrogen receptor gene is linked and/or associated with age of menarche in different ethnic groups. J Med
Genet 2005; 42: 796-800.

3. Segal NL, Stohs JH. Resemblance for age at menarche in female twins reared apart and together. Hum Biol 2007; 79: 623-35.

4. Gajdos ZK, Hirschhorn JN, Palmert MR. What controls the timing of puberty? An update on progress from genetic investigation. Curr Opin Endocrinol Diabetes Obes 2009; 16: 16-24.

5. Gluckman PD, Hanson MA. Evolution, development and timing of puberty. Trends Endocrinol Metab 2006; 17: 7-12.

6. Arias-Valencia MM. Comportamiento reproductivo en las etnias de Antioquia-Colombia. Salud Pública Mex 2001; 43: 268-78.

7. Silva HP, Padez C. Secular trends in age at menarche among Caboclo populations from Pará, Amazonia, Brazil: 1930-1980. Am J Hum Biol 2006; 18: 83-92.

8. Malina RM, Selby HA, Buschang PH, Aronson WL, Wilkinson RG. Adult stature and age at menarche in Zapotec-speaking communities in the Valley of Oaxaca, Mexico, in a secular perspective. Am J Phys Anthropol 1983; 60: 437-49.

9. Delgado H, Hurtado E. Crecimiento físico y menarquia en adolescentes de Guatemala. Arch Latinoam Nutr 1990; 40: 503-17.

10. Da Silva E P, Pelloso SM, de Barros Carvalho MD, de Ornelas Toledo MJ. Exploração de fatores de risco para câncer de mama em mulheres de etnia Kaingáng, Terra Indígena Faxinal, Paraná, Brasil, 2008. Cad. Saúde Pública 2009; 25: 1493-500.

11. Dittmar M. Age at menarche in a rural Aymara speaking community located at high altitude in northern Chile. Mankind Quarterly 2000; 40: 381-94.

12. Hernández MI, Unanue N, Gaete X, Cassorla F, Codner E. Edad de la menarquia y su relación con el nivel socioeconómico e índice de masa corporal. Rev Med Chile 2007; 135: 1429-36.

13. Quevedo I, Flores M, Castillo M. Características reproductivas de las mujeres consultantes en servicios de urgencia general de la VIII Región. Rev Med Chile 2005; 133: 929-34.

14. Amigo H, Bustos P, Muzzo S, Alarcón AM, Muñoz S. Age of menarche and nutritional status of indigenous and non-indigenous adolescents in the Araucanía Region of Chile. Ann Hum Biol 2010; 37: 554-61.

15. Chang HS, Odongua N, Ohrr H, Sull JW, Nam CM. Reproductive risk factors for cardiovascular disease mortality among postmenopausal women in Korea: the Kangwha Cohort Study, 1985-2005. Menopause 2011 (En prensa) Doi: 10.1097/gme.0b013e31821adb43.

16. Pandey M, Shukla VK. Lifestyle, parity, menstrual and 
reproductive factors and risk of gallbladder cancer. Eur J Cancer Prev 2003; 12: 269-72.

17. Travis RC, Key TJ. Oestrogen exposure and breast cancer risk. Breast Cancer Res 2003; 5: 239-47.

18. Xu WH, Xiang YB, Ruan ZX, Zheng W, Cheng JR, Dai Q et al. Menstrual and reproductive factors and endometrial cancer risk: Results from a population-based casecontrol study in urban Shanghai. Int J Cancer 2004; 108: 613-9.

19. Shrestha A, Olsen J, Ramlau-Hansen CH, Bech BH, Nohr EA. Obesity and age at menarche. Fertil Steril 2011: 2732-4.

20. Correia LL, Silveira DM, Silva AC, Campos JS, Machado MM, Rocha HA, et al. Prevalence and determinants of obesity and overweight among reproductive age women living in the semi-arid region of Brazil. Ciênc Saúde Coletiva 2011; 16: 133-45.

21. Harris MA, Prior JC, Koehoorn M. Age at menarche in the Canadian population: secular trends and relationship to adulthood BMI. J Adolesc Health 2008; 43: 54854.

22. Ministerio de Salud, Gobierno de Chile. Encuesta Nacional de Salud ENS Chile 2009-2010 [Documento electrónico] p. 111. Consultado en: 12/10/2011. Disponible en: http://www.minsal.gob.cl/portal/docs/page/ minsalcl/g_home/submenu_portada_2011/ens2010.pdf

23. Organización Internacional del Trabajo. Clasificación Internacional Uniforme de Ocupaciones. Consultado en 23/09/2011. Disponible en : http://www.ilo.org/public/ spanish/bureau/stat/isco/isco88/major.htm.

24. Instituto Nacional de Estadística, Gobierno de Chile. Remuneraciones y costos medios, Informe anual 2009. [Documento Electrónico] p. 29-31. Consultado en: 23/09/2011. Disponible en: http://www.ine.cl/canales/ menu/publicaciones/calendario_de_publicaciones/ pdf/080311/rem_09080311.pdf

25. Amigo H, Bustos P (Eds). Apellidos Mapuche, historia y significado. Santiago de Chile: Impresos Maigret Ltda; 2009.

26. Mosteller F, Gilbert JP, McPeek BM. Assessing time. to-event as an endpoint. En Lang TA, Secic M. How to report statistics in Medicine. $2^{\circ}$ Ed. Philadelphia, USA: ACP; 2006. p. 121.

27. Beyene Y, Martin MC. Menopausal experiences and bone density of Mayan women in Yucatan, Mexico. Am J Hum Biol 2001; 13: 505-511.

28. Arias-Valencia M M. Determinantes próximos de la fecundidad: comportamiento reproductivo de las indígenas Chamibida de Antioquia, Colombia. Cad. Saúde Pública 2005; 21: 1087-98.

29. PNUD, Universidad de La Frontera, MIDEPLAN. El
Índice de Desarrollo Humano en la población mapuche en la región de La Araucanía [Documento electrónico] 2003;8:18-19 [Consultado: 04/06/2011] En http://www. desarrollohumano.cl/otraspub/pub08/pub08.pdf

30. Frisch RE, McArthur JW. Menstrual cycles: fatness as a determinant as a minimum weight for height necessary for their maintenance or onset. Science 1974; 185: 94951.

31. Himes JH, Obarzanek E, Baranowski T, Wilson DM, Rochon J, McClanahan BS. Early Sexual Maturation, Body Composition, and Obesity in African-American Girls. Obes Res 2004; 12 Suppl: S64-72.

32. Gama A. Age at menarche in Portuguese rural women from Oleiros. Ann Hum Biol 2008; 35: 639-55.

33. Thomas F, Renaud F, Benefice E, De Meeüs T, Guegan JP. International Variability of Ages at Menarche and Menopause: Patterns and Main Determinants. Hum Biol 2001; 73: 271-90.

34. Larrea C, W Freire. Social inequality and child malnutrition in four Andean countries, Rev Panam Salud Pública 2002; 11: 356-64.

35. Malina RM, Pena Reyes ME, Tan SK, Little BB. Secular change in age at menarche in rural Oaxaca, southern Mexico: 1968-2000. Ann Hum Biol 2004; 31: 634-46.

36. JUNAEB. Mapa nutricional 2007 en adelante [En línea] [Consultado: 04/07/2011] En:http://www.junaeb.cl/prontus_junaeb/site/artic/20100121/pags/20100121095039. html

37. Codner E, Unanue N, Gaete X, Barrera A, MookKanamori D, Bazaes R, et al. Cronología del desarrollo puberal en niñas escolares de Santiago: relación con nivel socio-económico e índice de masa corporal. Rev Med Chile 2004; 132: 801-8.

38. Mumby HS, Elks CE, Li S, Sharp SJ, Khaw KT, Luben $\mathrm{RN}$ et al. Mendelian randomisation study of childhood BMI and early menarche. J Obes 2011 [en línea] doi:10.1155/2011/180729.

39. Amigo H, Bustos P, Erazo M, Radrigán ME. Comparación del estado nutricional de escolares indígenas y no indígenas. Rev Med Chile 1999;127: 903-910.

40. Bustos P, Muñoz S, Vargas C, Amigo H. Evolution of the nutritional situation of indigenous and non-indigenous Chilean schoolchildren. Ann Hum Biol 2009; 36: 298307.

41. Ossa X, Muñoz S, Amigo H, Bangdiwala SI. Secular trend in age at menarche in indigenous and non-indigenous women in Chile. Am J Hum Biol 2010; 22: 688-94.

42. Ersoy B, Balkan C, Gunay T, Egemen A. 2005. The factors affecting the relation between the menarcheal age of mother and daughter. Child Care Health Dev 31: 303-8.

43. Chang S, Chen K. Age at menarche of three-generation 
families in Taiwan. Ann Hum Biol 2008; 35: 394-405.

44. PNUD, MIDEPLAN. Las trayectorias del desarrollo humano en las comunas de Chile (1994-2003) [Documento electrónico] 11: 89-90 [Consultado: 02/07/2011] En http: //desarrollohumano.cl/otraspub/pub12/IDHC\%20 con\%20portada.pdf

45. Sanhueza P. Experiencia de desarrollo humano en la IX Región de La Araucanía, Chile. [Documento electrónico] Noésis. Revista de Ciencias Sociales y Humanidades
2005; 15: 131-58 [Consultado:04/07/2011] En http:// redalyc.uaemex.mx/pdf/859/85902706.pdf

46. Gaete X, Codner E. Adelanto de la pubertad en Chile y el mundo. Rev Chil Pediatr 2005; 77: 456-65.

47. Programa de las Naciones Unidas para el Desarrollo (PNUD) Desarrollo Humano en Chile. Las nuevas tecnologías: ¿un salto al futuro? 2006 [Documento electrónico] p. 213-24 [Consultado: 06/04/2012] En http:// http://www.centrodesarrollohumano.org/ 